\title{
Post-coronavirus disease 2019 children in Sarajevo-Lessons learnt?
}

\author{
Senka Mesihović-Dinarević* , Timur Šečić \\ Department of pediatrics, Eurofarm Polyclinic Sarajevo, Sarajevo, Bosnia and Herzegovina
}

\begin{abstract}
Introduction: A new disease coronavirus disease 2019 (COVID-19) is with insufficiently known epidemiological characteristics and spectrum of clinical expression in childhood. Children have a lower incidence of this disease with a predominance of mild forms but severe clinical forms, such as among others, acute respiratory distress syndrome, and multisystem inflammatory syndrome may occur, according to current findings. In children with atypical symptomatology and positive or suspicious epidemiological survey, practitioners should consider the possibility of COVID-19.

Methods: This study formed the group of 70 children previously healthy or with no pre-existing heart disease from Sarajevo with positive post-COVID history. Following the history of disease and epidemiological data, establishing the $1^{\text {st }}$ day of disease or contact, a detailed cardiovascular examination was performed, including parameters of body weight, height, oxygen saturation, pulse, blood pressure, 12 leads electrocardiogram (ECG) done on Schiller machine, values of polymerase chain reaction (PCR), or serological test on corona: Immunoglobulin (Ig) G and IgM. Echocardiographic examination was done using $\mathrm{M}, \mathrm{B}$ mode, color, continuous wave, and pulse wave Doppler in standard views. Laboratory blood tests included: Full blood count, creatinine phosphokinase myofibril, creatinine phosphokinase, lactate dehydrogenase; liver enzymes, D dimer, C reactive protein, and urine.

Results: Majority of children (64.3\%) were asymptomatic. ECG was normal in relation to patients' age except in eight patients (intermittent palpitations on exertion) who had short PR interval 0.120-0.140 ms, with no delta wave, with heart rate within the normal range according to age, so $24 \mathrm{~h} \mathrm{ECG} \mathrm{Holter} \mathrm{was} \mathrm{performed} \mathrm{without} \mathrm{any} \mathrm{significant} \mathrm{arrhythmias,}$ incomplete right branch block has been documented in 12\%, monofocal ventricular ectopic extrasistoly in $15 \%$. Mean IgG, as a marker of infection, showed a statistical significance when compared between age Groups I and II $(<5)$ and older groups: III, IV, and V $(>5)(p<0.05 ; p=0.043)$. PCR test was negative in 9 (70 children), although they showed symptoms, COVID-19 infection clinical data, and positive laboratory findings. Echocardiogram was normal in all patients with normal ejection fraction of the left ventricle.

Conclusion: The possibility of COVID-19 in children with atypical symptomatology and positive or suspicious epidemiological survey should be in the focus of every pediatrician at primary care institutions nowadays. Cardiovascular assessment should always be an option in post-COVID patients. Immunological assessment is necessary in post-COVID patients in order to gain a further understanding of PTS status. With more serological testing for severe acute respiratory syndrome coronavirus 2 physicians would be able to make a diagnosis of COVID-19 timely and more accurately, as well as to evaluate the role of asymptomatic children in disease transmission and to assess the importance of protective antibodies and the distribution of COVID-19.
\end{abstract}

Keywords: Coronavirus disease 2019; post-coronavirus disease 2019, children

\section{INTRODUCTION}

The exact incidence rate of severe acute respiratory syndrome coronavirus 2 (SARS-CoV-2) infections in children is not known due to lack of widespread testing and the prioritization of testing adults and those with severe forms of illness. Hospitalization rates in children are significantly lower than hospitalization rates in adults with coronavirus

${ }^{*}$ Corresponding author: Senka Mesihović-Dinarević, Department of pediatrics, Eurofarm Polyclinic Sarajevo, Sarajevo, Bosnia and Herzegovina. E-mail: dsenka@bih.net.ba

Submitted: 04 May 2021/Accepted: 09 June 2021

DOI: https://doi.org/10.17532/jhsci.2021.1331 disease 2019 (COVID-19), suggesting that children may have less severe manifestations of illness from COVID-19 compared to adults $(1,2)$. Recent evidence suggests that compared to adults, children are likely to have similar viral loads in their nasopharynx (3), similar secondary infections rates, and can spread the virus to others $(4,5)$. Severe forms of the disease are more pronounced in infants versus children $11-15$ years and $\geq 16$ years ( $11 \%$ vs. $4 \%$ and $3 \%$ ) and it is estimated that $\geq 90 \%$ of children have asymptomatic, mild, or moderate forms of COVID-19 (6). The primary target for SARS-CoV-2 is the respiratory tract, but cardiovascular involvement is emerging as one of the most 
significant and life-threatening complications of SARSCoV-2 infection in adults, possibly in children as well (7).

The aim of this study is to evaluate the knowledge in postCOVID children in Sarajevo, about SARS-CoV-2, epidemiology, transmission, and clinical presentation including immunological and cardiovascular status.

\section{METHODS}

This study formed the group of 70 children previously healthy or with no pre-existing heart disease from Sarajevo with positive post-COVID history. They were referred from local pediatrician or by parents concerned due to child's symptoms, for cardiovascular assessment by pediatric cardiologist. Patients were evaluated in Eurofarm Polyclinic in Sarajevo, during period October 2020 till April 2021. Ethical board of polyclinic Eurofarm gave a approval for study.

Since the respondents were children, written consent was obtained from their parents/guardians to participate in the study.

Following the history of disease and epidemiological data, establishing the $1^{\text {st }}$ day of disease or contact, a detailed cardiovascular examination was performed, including parameters of body weight, height, oxygen saturation, pulse, blood pressure (BP), 12 leads electrocardiogram (ECG) done on Schiller machine, values of polymerase chain reaction (PCR), or serological test on corona: Immunoglobulin (Ig) $\mathrm{G}$ and Ig M. In all patients the echocardiography had been performed which provided data on the morphology and hemodynamic of the heart with special attention to myocardium, ejection fraction of left ventricle, morpho hemodynamics of coronary blood vessels possible dilatation, that is, aneurysms of coronary blood vessels and signs of myocardial lesions. Echocardiographic examination was done on ultrasound machine Toshiba Aplio 300 using M, B mode, color, continuous wave, and pulse wave Doppler in standard views. Laboratory blood tests included: Full blood count (FBC), creatinine phosphokinase myofibril (CPKMB), creatinine phosphokinase (CPK), lactate dehydrogenase (LDH); liver enzymes, $\mathrm{D}$ dimer, $\mathrm{C}$ reactive protein (CRP), and urine.

The SPSS program (Version 26.0.0.0.2019) has been applied in this study.

\section{RESULTS}

In the group of 70 children ( 40 boys) there were: Infants (under 1 years of age):10, 1-5 years: 20; 6-10 years: 12; 11-15 years: 21 ; and $16-18$ years: 7 ; forming, five groups (Figures 1 and 2).

All children were in relation to percentile scale within the average height and weight range from $25 \mathrm{p}-97 \mathrm{p}$. The mean body temperature at the time of examination was: $36.6^{\circ} \mathrm{C}$ $\left(34.3-37.6^{\circ} \mathrm{C}\right)$, mean oxygen saturation was $97.6 \%$ (94$99 \%$ ). Pulse rate was within a normal range for age as was BP for age, height, and sex.

Before referral to pediatric cardiologists, 24 patients were seen by local pediatrician. The period from contact with COVID-19 through a family member or at school, until referral to pediatrician was 7.5 days (1-21 days).

Symptoms differ depending on age group; younger children had no or mild symptoms in comparison to the older group of children (Table 1).

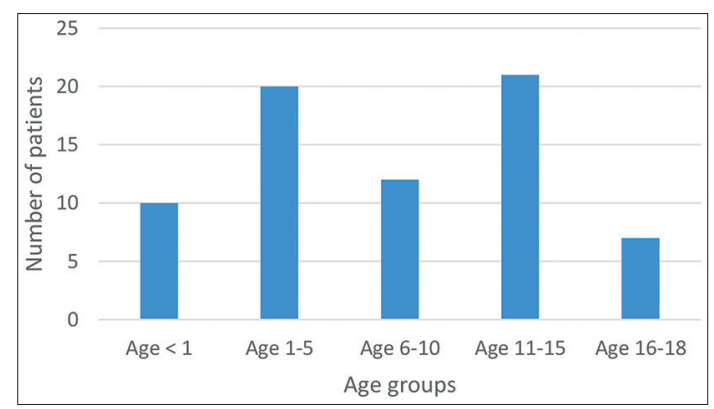

FIGURE 1. Age distribution.

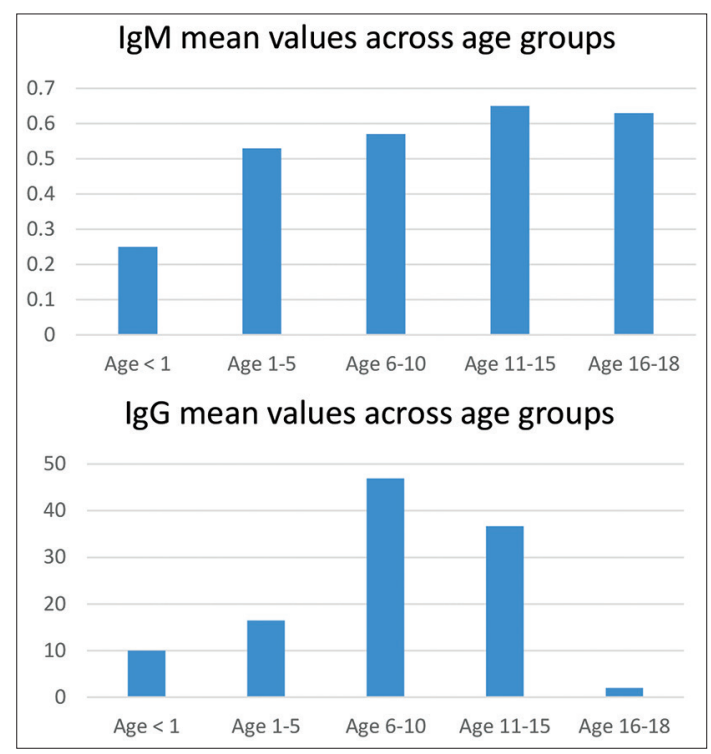

FIGURE 2. IgM and $\lg G$ mean values.

Majority of children (64.3\%) were asymptomatic, except two 14-year-old boys, with palpitation on exertion, shortness of breath, including ECG changes: Denivelation of ST segment in lead II, V5 and V6, lower oxygen saturation $91 \%$ and $94 \%$, elevated CPKMB (38 and 45 (with mildly enlarged in one patient diameter of left coronary artery (LCA) up to $3.8 \mathrm{~mm}$, no aneurysm, no skin changes, and with normal EF of LV. They were on short period (10-15 days) nonsteroidal treatment, including low doses of Aspirin and vitamins ( $C$ and $D)$, rest and no sport activities. After treatment and non-activity regime, they were fully recovered, free of symptoms, with normal oxygen saturation and normal values of CPKMB and $\mathrm{FBC}$, diameter of LCA was within the normal range according to age and body weight of patient.

FBC including platelets and white blood count were within normal range, as well as urine, $\mathrm{D}$ dimer, $\mathrm{CRP}, \mathrm{LDH}$, liver enzymes, and CPK; CPKMB except as mentioned earlier.

ECG was normal in relation to patients' age except in eight patients (intermittent palpitations on exertion) who had short PR interval $0.120-0.140 \mathrm{~ms}$, with no delta wave, with heart rate within the normal range according to age, so $24 \mathrm{~h}$ ECG Holter was performed without any significant arrhythmias, incomplete right branch block has been documented in $12 \%$, and monofocal ventricular ectopic extrasistoly in $15 \%$ (Table 2).

IgG (Group I and II) versus IgG (Group III, IV, and V) $p<0.043$. 
Mean IgG, as a marker of infection, showed a statistical significance when compared between age Groups I and II $(<5)$ and older Groups: III, IV, and V $(>5) .(p<0.05 ; p=0.043)$. PCR test was negative in $9 / 70$ children, although they showed symptoms, COVID-19 infection clinical data, and positive laboratory findings.

Echocardiogram was normal in all patients with normal ejection fraction of left ventricle. No pericardial effusion, vegetations or thrombus was detected. Mean diameter of coronary arteries right (RCA) and left (LCA) ranged $1.98 \mathrm{~mm}$ to LCA $2.09 \mathrm{~mm}$. Z score calculation for coronary arteries including: body weight, height and gender of every patient, was within a normal values for RCA and LCA $(8,9)$. No anomalies were detected in their flow and diameter, with no aneurisms, except stated earlier in the text (Figure 3).

\section{DISCUSSION}

The gold standard for diagnosis COVID-19 in children is PCR test, therefore in children with mild form of corona or who have been in contact with an infected person, a serology test of the Corona titer with $\operatorname{IgG}$ and $\operatorname{IgM}$ was conducted. The interpretation of Corona serological test is: A positive IgM titer (active process) can last for weeks and sometimes even months. An indication of the past infection

TABLE 1. Clinical features in children with confirmed COVID-19 contact

\begin{tabular}{lccccc}
\hline Symptoms & Group 1 & Group 2 & Group 3 & Group 4 & Group 5 \\
\hline Asymptomatic & 8 & 14 & 8 & 12 & 3 \\
Cough & 2 & 5 & 3 & 5 & 0 \\
Tiredness & 0 & 0 & 1 & 1 & 0 \\
Sweats & 0 & 0 & 0 & 2 & 3 \\
Palpitation on & 0 & 2 & 1 & 4 & 1 \\
exertion & & & & & \\
Abdominal pain & 0 & 1 & 1 & 2 & 0 \\
Fever & 2 & 6 & 4 & 7 & 2 \\
Diarrhea & 0 & 1 & 1 & 2 & 0 \\
Exanthema & 1 & 0 & 1 & 0 & 1 \\
\hline
\end{tabular}

TABLE 2. Laboratory data in children with confirmed COVID-19 contact

\begin{tabular}{lccccc}
\hline Ig: Immunoglobulin & Group 1 & Group 2 & Group & Group & Group 5 \\
& & & 3 & 4 & \\
\hline Corona IgG & 10.02 & 16.48 & 46.92 & 36.73 & 1.98 \\
Corona IgM & 0.25 & 0.53 & 0.57 & 0.65 & 0.63 \\
\hline
\end{tabular}

is a positive IgG titer, meaning the child was in contact with the virus or was suffering from COVID-19. According to the recent literature research, children have a lower incidence of the disease with a predominance of mild forms of the disease, which is the case in our study group $(10,11)$.

Over $90 \%$ patients ranged from asymptomatic to moderate in the largest pediatric population-based study to date with 2143 cases. However, the proportion of severe and critical cases was $10.6 \%$ under 1 year of age, compared to $7.3,4.2$, 4.1 , and $3.0 \%$ among the $1-5,6-10,11-15$, and $>15$ years subsets, suggesting that infants may be at higher risk of severe respiratory failure than initially thought (12).

COVID-19 is most manifested as mild disease in children according to the literature and data in our study. Compared to adults, it is believed that children have a qualitatively different response to SARS-CoV-2, which may be related to the characteristics of their immune system to better control and localize COVID infection to the upper respiratory tract resulting in milder cold-like infections (13-15). It is likely that the simultaneous presence of common strains of coronavirus and other respiratory viruses in the lung and airway mucosa, which are common in children, may limit the growth of SARS-CoV-2 due to direct interaction, competition, and cross-immune protection (15). A more accurate response to the distribution and course of COVID-19 infection in neonates and young children, who have not been exposed to common coronaviruses and other respiratory viruses, and some viral infections are known to result in more severe respiratory infections and respiratory failure, what the future research would be able to provide.

A strong innate immune response, immune training as a possible protection factors $(16,17)$, which may be associated with previously given BCG and MMR vaccines, as well as previous exposure to respiratory viruses, can lead to lower incidence and mostly mild forms of COVID-19 in children. There are changes in innate and adaptive immunity with aging, and adults are more susceptible to severe infections and even severe forms of COVID-19 due to the large release of cytokines (cytokine storm) from macrophages, monocytes, and lymphocytes, leading to lung tissue damage and multiorgan failure (16-18). Alveolar epithelial cells in children have better regenerative potential, and most children do not have certain risk factors (smoking and obesity) and comorbidities like adults, which may play a role in the greater expression of more severe clinical forms of COVID-19 in adults $(13,18)$.
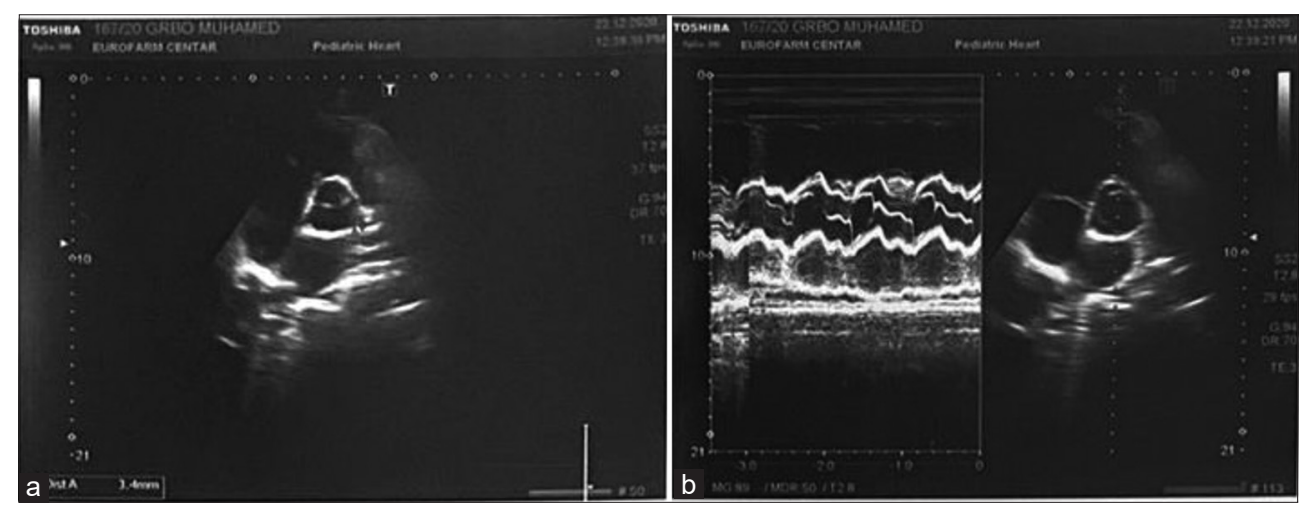

FIGURE 3. (a): 2D echocardiogram, short axis view, level of aorta, showing dilated diameter of left coronary artery, and b: $M$ and $B$ mod view of the same patient showing normal opening of three leaflet coronary artery. 
The use of reverse transcription PCR (RT-PCR) from nasopharyngeal or throat swabs, and in suspected cases with initial negative findings of RT-PCR, from nasopharyngeal swabs, lower airway aspirate (16) may be used according to present symptomatology, as well as clear or suspicious epidemiological survey. Having in mind that most children are asymptomatic or have mild symptoms, and no RT-PCR is performed, this may play a role in assessing the prevalence of COVID-19 infection in children.

In the past several months, a few children have been identified with a developed multisystem inflammatory response to SARS-COV-2, in common with Kawasaki disease, staphylococcal and streptococcal toxic shock syndrome, bacterial sepsis, and macrophage activation syndrome (18). A generalized febrile illness, Kawasaki disease, in children $<5$ years characterized by fever, skin rash, lymphadenopathy, and conjunctivitis. Inflammation of the coronary arteries occurs in severe cases of Kawasaki disease that spreads, causing a coronary artery aneurysm with the possibility of myocardial infarction in children, which is verified by ultrasound color Doppler examination of the heart (19-22). Kawasaki is not an infectious but a reactive disease, which is treated with immunoglobulins, corticosteroids, and aspirin (15). In our study group of children there were no cases of multisystem inflammatory response to SARSCOV-2. In two children (7 and 2-year-old boys) Kawasaki-like hyper inflammatory syndrome as a delayed response to SARSCOV-2 infection, which manifested in, among other things, mucocutaneous changes, elevated markers of inflammation, lymphopenia, thrombocytopenia, and complement consumption with myocarditis, has been described by Licciardi et al. (23). Titers of IgG and IgM on the SARS-CoV-2 virus were high but PCR test for SARS-CoV-2 was negative. Patients were successfully treated with intravenous immunoglobulin.

Pediatric multisystem inflammatory syndrome (PMIS) affects mostly previously healthy school-aged children and adolescents presenting with Kawasaki disease-like features and multiple organ failure with a focus on the heart, accounting for most cases of pediatric COVID-19 mortality (7), according to meta-analysis of 16 studies with size $>10$ patients with complete data about cardiovascular involvement in children with PMIS. They frequently presented cardiogenic shock (53\%), ECG alterations (27\%), myocardial dysfunction (52\%), and coronary artery dilation (15\%). Most cases required PICU admission (75\%) and inotropic support (57\%), with the rare need for extracorporeal membrane oxygenation (4\%). Although rare deaths have been reported (2\%), almost all these children recovered fully within a few days.

The evidence of cardiac manifestation in previously healthy children without PMIS has also been reported. A few cases have been reported since the beginning of the pandemic, with cardiovascular involvement like adults, in the form of myocarditis, pericarditis, heart failure, cardiogenic shock, cardiac arrhythmia, and pulmonary hypertension $(24,25)$. These cases are usually reported as severe or critically ill patients, although infrequent, pointing out the relevance of the cardiac involvement also in previously healthy children with COVID-19.

In our study, the values of immunoglobulin $G$ were significantly higher in the older group of children with $\mathrm{p} 0.043$ indicating that with age the immune system is getting more responsive to the virus. There are changes in innate and adaptive immunity with aging, and adults are more susceptible to severe infections and even severe forms of COVID-19 due to the large release of cytokines (cytokine storm) from macrophages, monocytes, and lymphocytes, leading to lung tissue damage and multiorgan failure $(13,14)$.

It was found analyzing the symptoms in children that, among other things, fever is present in $40-60 \%$ of children, cough occurs in $40-50 \%$ of children, and headache, myalgia, and sore throat are more common than shortness of breath. After 7-10 days (26), usually children recover, and more severe forms are characterized by dyspnea, hypoxia, cyanosis, dehydration with signs of respiratory distress syndrome, shock, myocardial lesions, or multiorgan failure (27-29). In our study group, fever was present in 15 (70), cough 21 (70), shortness of breath 8 (70) patients and majority were asymptomatic, which is the case with majority of reported pediatric patients in post-COVID period.

Identical changes have been reported by Andina et al. (30) in Madrid in 22 children, aged 6-17 years with moderate symptoms of COVID-19 disease. These atypical manifestations of COVID-19 disease did not require treatment and had a good prognosis.

In the context of pediatric COVID-19, there are little data about the occurrence of arrhythmias. Data from two smallsized studies showed that hospitalized pediatric COVID19 cases could present a rate of cardiac arrhythmia as high as $16-20 \%(31,32)$. Initial data from Chinese observations suggest that contrary to adult patients, whose myocardial involvement is sometimes correlated with the appearance of life-threatening arrhythmias, children with COVID-19 share less harmful rhythm troubles, such as supraventricular tachycardia, premature atrial and ventricular complexes, first-degree atrioventricular blocks, and incomplete right bundle branch block which is in correlation with data from our post-COVID group of children; no significant rhythm disturbances. The coexistence of myocardial injury and cardiac arrhythmias with COVID-19 makes it challenging to diagnose and manage this specificity. The early recognition of cardiac symptoms and their timely treatment may be of pivotal importance to improve the prognosis of pediatric patients, overall, in those with severe disease.

There is still scarce data about the role of cardiovascular involvement in COVID-19 in children. Based on our review and recent literature data, the cardiovascular involvement seems to be a relevant factor of SARS-CoV-2 infection in children. The performance of a cardiac workup and close cardiovascular monitoring of children with severe SARS-CoV-2 infection is highly recommended.

\section{Limitations of the study}

- Due to random patients' selection for the study, the number of the patients in the group varies

- The referral to pediatric cardiologist differs due to incoherent protocol of post-COVID assessment.

\section{CONCLUSION}

The possibility of COVID-19 in children with atypical symptomatology and positive or suspicious epidemiological survey, should be in the focus of every pediatrician at primary care institutions nowadays. Cardiovascular assessment should always be an option in post-COVID patients. 
Immunological assessment is necessary in post-COVID patients to gain a further understanding of PTS status. With more serological testing for SARS-CoV-2 physicians would be able to make a diagnosis of COVID-19 timely and more accurately, as well as to evaluate the role of asymptomatic children in disease transmission and to assess the importance of protective antibodies and the distribution of COVID19. According to the instructions of the Centre for Disease Control, it is necessary to continue practicing physical distancing, washing hands, and of course wearing masks.

\section{DECLARATION OF INTERESTS}

Authors declare no conflict of interest.

\section{REFERENCES}

1. Bixler D, Miller AD, Mattison CP, Taylor B, Komatsu K, Pompa XP, et al. SARS-CoV2-associated deaths among persons aged $<21$ years United States, February 12-July 31, 2020. MMWR Morb Mortal Wkly Rep 2020;69(37):1324-9.

https://doi.org/10.15585/mmwr.mm6946a7.

2. Leeb RT, Price S, Sliwa S, Kimball A, Szucs L, Caruso E, et al. COVID-19 trends among school-aged children United States, March 1-September 19, 2020. MMWR Morb Mortal Wkly Rep 2020;69:1410-5.

https://doi.org/10.15585/mmwr.mm6939e2.

3. Sargent TH, Muller WJ, Zheng X, Rippe J, Patel AB, Kociolek LK. Age-related differences in nasopharyngeal severe acute respiratory syndrome coronavirus 2 (SARSCoV-2) levels in patients with mild to moderate coronavirus disease 2019 (COVID-19). JAMA Pediatrics 2020;174(9):902-3.

https://doi.org/10.1001/jamapediatrics.2020.3651.

4. Yonker LM, Neilan AM, Bartsch Y, Patel AB, Regan J, Arya P, et al. Pediatric severe acute respiratory syndrome coronavirus 2 (SARS-CoV-2): Clinical presentation, infectivity, and immune responses. J Pediatr 2020;227:45-52.e5. https://doi.org/10.1016/j.jpeds.2020.08.037.

5. Laws RL, Chancey RJ, Rabold EM, Chu VT, Lewis NM, Fajans M, et al. Symptoms and transmission of SARS-CoV-2 among children Utah and Wisconsin, March-May 2020. Pediatrics 2020;146(6):e2020027268

6. DeBiasi RL, Song X, Delaney M, Bell M, Smith K, Pershad J, et al. Severe coronavirus disease-2019 in children and young adults in the Washington, DC, metropolitan region. J Pediatr 2020;223:199-203.e1.

https://doi.org/10.1016/j.jpeds.2020.05.007

7. Rodriguez-Gonzalez M, Castellano-Martinez A, Cascales-Poyatos HM, PerezReviriego AA. Cardiovascular impact of COVID-19 with a focus on children: A systematic review. World J Clin Cases 2020;8:5250-83.

https://doi.org/10.12998/wjcc.v8.i21.5250

8. Kobayashi T, Fuse S, Sakamoto N, Mikami M, Ogawa S, Hamaoka K, et al. New $Z$ score curve of the coronary arterial internal diameter using the lambda-mu-sigma method in a pediatric population. J Am Soc Echocardiogr 2016;29(8):794-801.e29. https://doi.org/10.1161/circimaging.117.006979.

9. Lopez L, Colan S, Stylianou M, Granger S, Trachtenberg F, Frommelt P, et al. Relationship of echocardiographic $Z$ scores adjusted for body surface area to age, sex, race, and ethnicity: The pediatric heart network normal echocardiogram database. Circ Cardiovasc Imaging 2017;10(11):e006979.

10. Mesihovic-Dinarevic S. COVID-19 and children. J Health Sci 2021;11(1):59-9.

11. Sporišević L, Mesihović-Dinarević S, Jogunčić A, Pilav A. Coronavirus disease 2019 (COVID-19): A new pediatric challenge. J Health Sci 2020;10:103-8. https://doi.org/10.17532/jhsci.2020.972

12. de Rose DU, Piersigilli F, Ronchetti MP, Santisi A, Bersani I, Dotta A, et al. Nove coronavirus disease (COVID-19) in newborns and infants: What we know so far. Ital J Pediatr 2020;46(1):56 https://doi.org/10.1186/s13052-020-0820-x.

13. Dhochak N, Singhal T, Kabra SK, Lodha R. Pathophysiology of COVID-19: Why children fare better than adults? Indian J Pediatr 2020;87(7):537-46

https://doi.org/10.1007/s12098-020-03322-y.

14. Brodin P. Why is COVID-19 so mild in children? Acta Paediatr 2020;109(6):1082-3 https://doi.org/10.1111/apa.15271

15. Lee PI, Hu YL, Chen PY, Huang YC, Hsueh PR. Are children less susceptible to COVID-19? J Microbiol Immunol Infect 2020;53(3):371-2.

16. Nickbakhsh S, Mair C, Matthews L, Reeve R, Paul C, Johnson PC, et al. Virus-virus interactions impact the population dynamics of influenza and the common cold. Proc Natl Acad Sci USA 2019;116(52):27142-50.

https://doi.org/10.1073/pnas.1911083116.

17. Pavone P, Ceccarelli M, Taibi R, La Rocca G, Nunnari G. Outbreak of COVID-19 infection in children: Fear and serenity. Eur Rev Med Pharmacol Sci 2020;24(8):4572-5.

18. Royal College of Pediatrics and Child Health. Guidance-Paediatric Multisystem Inflammatory Syndrome Temporally Associated with COVID-19; 2020. Available from: https://www.rcpch.ac.uk/resources/guidance-paediatric-multisystem-inflammatory-syndrome-temporally-associated-covid-19. [Last accessed on 2020 May 17]. https://doi.org/10.1007/s13312-020-1975-7.

19. Viner RM, Whittaker E. Kawasaki-like disease: Emerging complication during the COVID-19 pandemic. Lancet 2020;395(10239):1741-3 https://doi.org/10.1016/s0140-6736(20)31129-6.

20. Mesihović-Dinarević S. Paediatric Ultrasound Atlas of Heart Anomalies. $2^{\text {nd }}$ ed. Sarajevo: Bookline; 2017

21. Dinarević S, Henein MY, Zaghal AA, Shinebourne EA, Gibson DG. Exercise stress echocardiography in children with Kawasaki disease-left ventricular axis selectively impaired. The second world congress of paedatric cardiology and cardiac surgery. Am J Cardiol 1998;81(11):1356-9.

https://doi.org/10.1016/s0002-9149(98)00168-4.

22. Henein MY, Dinarević S, O'Sulliven C, Gibson DG, Shinebourne EA. Exercise echocardiography in children with Kawasaki disease: Ventricular long axis is selectively abnormal. Am J Cardiol 1998;81(11):1356-9.

https://doi.org/10.1016/s0002-9149(98)00168-4.

23. Licciardi F, Pruccoli G, Denina M, Parodi E, Taglietto M, Rosati S, et al. SARS-CoV 2-induced Kawasaki-like hyperinflammatory syndrome: A novel COVID phenotype in children. Pediatrics 2020;146(2):e20201711. https://doi.org/10.1542/peds.2020-1711.

24. Rodriguez-Gonzalez M, Rodríguez-Campoy P, Sánchez-Códez M, Gutiérrez-Rosa I, Castellano-Martinez A, Rodríguez-Benítez A. New onset severe right ventricular failure associated with COVID-19 in a young infant without previous heart disease. Cardiol Young 2020;30(9):1346-9

https://doi.org/10.1017/s1047951120001857.

25. Cui $Y$, Tian $M$, Huang $D$, Wang $X$, Huang $Y$, Fan L, et al. A 55-day-old female infant infected with 2019 novel coronavirus disease: Presenting with pneumonia, Liver injury, and heart damage. J Infect Dis 2020;221(11):1775-81.

https://doi.org/10.1093/infdis/jiaa113.

26. Zimmermann P, Curtis N. Coronavirus infections in children including COVID-19: An overview of the epidemiology, clinical features, diagnosis, treatment and prevention options in children. Pediatr Infect Dis J 2020;39(5):355-68.

https://doi.org/10.1097/inf.0000000000002660

27. CDC COVID-19 Response Team. Coronavirus disease 2019 in children-United States, February 12-April 2, 2020. MMWR Morb Mortal Wkly Rep 2020;69(14):422-6. https://doi.org/10.15585/mmwr.mm6914e4.

28. Dong $Y$, Mo X, Hu Y, Qi X, Jiang F, Jiang Z, et al. Epidemiological characteristics of 2143 pediatric patients with 2019 Coronavirus disease in China. Pediatrics 2020;58(4):712-3.

29. Sankar J, Dhochak N, Kabra SK, Lodha R. COVID-19 in children: Clinical approach and management. Indian J Pediatr 2020;87(6):433-42. https://doi.org/10.1007/s12098-020-03292-1.

30. Andina D, Noguera-Morel L, Bascuas-Arribas M, Gaitero-Tristán J, Alonso-Cadenas JA, Escalada-Pellitero S, et al. Chilblains in children in the setting of COVID-19 pandemic. Pediatr Dermatol 2020;37(3):406-11. https://doi.org/10.1111/pde.14215

31. Samuel S, Friedman RA, Sharma C, Ganigara M, Mitchell E, Schleien C, et al. Incidence of arrhythmias and electrocardiographic abnormalities in symptomatic pediatric patients with PCR-positive SARS-CoV-2 infection, including drug-induced changes in the corrected QT interval. Heart Rhythm 2020;17(11):1960-6. https://doi.org/10.1016/j.hrthm.2020.06.033.

32. Xia W, Shao J, Guo Y, Peng X, Li Z, Hu D. Clinical and CT features in pediatric patients with COVID-19 infection: Different points from adults. Pediatr Pulmonol 2020;55(5):1169-74 https://doi.org/10.1002/ppul.24718.

\section{RELATED ARTICLES PUBLISHED IN JHSCI}

1. Sporišević L, Mesihović-Dinarević S, Jogunčić A, Pilav A. Coronavirus disease 2019 (COVID-19): a new pediatric challenge. JHSCI. 2020;10(2):103-8. 